\title{
EL USO DE LAS TECNOLOGÍAS DE INFORMACIÓN COMO MEDIO DE ALFABETIZACIÓN EN LAS INSTITUCIONES DE EDUCACIÓN DE HONDURAS
}

\author{
ISSN 2219-6722 \\ ISSNE 2222-2707 \\ Nelson Roberto Raudales García, Universidad Nacional Autónoma de Honduras (UNAH), \\ Facultad de Ciencias Económicas, Administrativas y Contables (FCEAC), \\ Instituto de Investigaciones Económicas y Sociales (IIES), \\ Ciudad universitaria, edificio C2, primer piso, Tel/fax: (504) 2239-1849 \\ E-mail: nraudales@iies-unah.org
}

Revista Economía y Administración (E\&A)

\section{RESUMEN}

La era de las Nuevas Tecnologías de la información y comunicación (NTIC's) han influenciado de diversas formas en la vida de los seres humanos, hoy en día es inevitable su aplicación y uso de tales herramientas para el desarrollo de actividades médicas, científicas, investigación e innovación en las diversas áreas del saber. Por consiguiente el uso de tales herramientas en la transferencia de conocimientos no queda separado del desarrollo de actividades aplicables en las Instituciones de Educación Superior (IES) para lograr trascender en la vida económica de las personas y/o en la inclusión de medidas orientadas a mejorar las políticas públicas.

Asimismo, a nivel operativo de IES se vuelve imperante el reto que involucre a su personal en desempeñar el proceso de transformación y actualización académico-curricular docente, para lograr aprovechar al máximo tales propósitos sin desestimar en la medida de lo posible el establecimiento y aplicación de medios/políticas educativas que permitan a las IES incursionar eficientemente en tales iniciativas.

Además, el personal docente encargado de los procesos de enseñanza y/o transferencia de conocimientos debe disponer de las habilidades que les permitan trascender, o estar dispuestos a lograr la asimilación de tales herramientas para los fines requeridos ello para adaptarse de manera progresiva y constante a los cambios que dicho fenómeno globalizante conlleva.

Palabras Clave: Educación Superior, Nuevas Tecnologías de Información y Comunicación, Educación Virtual. 


\title{
THE USE OF INFORMATION TECHNOLOGY AS A MEANS OF LITERACY EDUCATION INSTITUTIONS OF HONDURAS
}

\author{
ISSN 2219-6722 \\ ISSNE 2222-2707 \\ Nelson Roberto Raudales García, Universidad Nacional Autónoma de Honduras (UNAH), \\ Facultad de Ciencias Económicas, Administrativas y Contables (FCEAC), \\ Instituto de Investigaciones Económicas y Sociales (IIES), \\ Ciudad universitaria, edificio C2, primer piso, Tel/fax: (504) 2239-1849 \\ E-mail: nraudales@iies-unah.org
}

\begin{abstract}
The era of new technologies of information and communication technologies (ICT's) have been influenced in various ways in the lives of human beings today is inevitable the use of such tools for the development of medical, scientific, research and innovation in various areas of knowledge. Therefore the use of such tools in the transfer of knowledge is not separate from the development of activities applicable in Higher Education Institutions (HEIs) in order to transcend the economic life of the people.
\end{abstract}

Also, operating at IES becomes prevailing challenge involving its staff in performing the transformation and upgrade teaching academic curriculum, in order to make the most of such purposes without underestimating the extent possible the establishment and media application / educational policies that enable HEIs enter efficiently in such initiatives.

Also, teachers responsible for teaching and transfer of knowledge must have the skills to transcend, or be prepared to achieve the uptake of such tools for their intended use it to fit in a progressive and constant changes globalizing phenomenon that entails.

Keywords: High Education, New Comunication and Information Technologies, Virtual Education.

Revista Economía y Administración (E\&A) 


\section{INTRODUCCIÓN}

Las Nuevas tecnologías de la información y las comunicaciones (NTIC's) son herramientas orientadas que pueden contribuir en el desarrollo socioeconómico de los países, cuya asimilación, apropiación e inclusión promueve aspectos de innovación, crecimiento económico, y la inclusión social, etc. Las NTIC's son una importante fuente de generación de información y conocimiento $\mathrm{y}$, como tal, se han constituido en pilares fundamentales para el desarrollo económico y social. Los constantes avances de estas tecnologías promueven innovaciones en todos los ámbitos de la economía y la sociedad, que se traducen en beneficios ligados no solo al crecimiento económico, sino también a la inclusión social, al facilitar la prestación de servicios como educación, salud, investigación y gestión gubernamental.

Aunado a ello, el desarrollo/existencia y creación de las plataformas e-learning, mismas que constituyen las bases educativas o espacios y entornos virtuales de enseñanza y aprendizaje, los que actualmente forman esta realidad tecnológica

Revista Economía y Administración (E\&A) creada en Internet y que da soporte a la enseñanza y el aprendizaje y transferencia de conocimientos particularmente en el área educativa. En estos momentos es plausible establecer que su uso ha transformando gran parte de los espacios de enseñanza tradicionales en espacios virtuales de enseñanza y aprendizaje. ${ }^{3}$

Entender el funcionamiento y apropiamiento de estas nuevas herramientas y saber cómo utilizarlas para mejorar la enseñanza y el aprendizaje pudiera convertirse en una tarea compleja y difícil; debido particularmente a que se vuelve necesario la realización de otras investigaciones las cuales deberán partir por identificar, diagnosticar y mapear si existe el recurso humano que disponga de las habilidades y destrezas necesarias para la asimilación, apropiamiento de estas plataformas para la trasferencia efectiva de conocimientos.

El desarrollo del presente artículo, describe lo que son las NTIC's, su funcionamiento $\mathrm{y}$, que aspectos pueden contribuir para mejorar la enseñanza y educación de forma tal que permita tener una visión clara de los conceptos para entender adecuadamente las plataformas virtuales y su aplicación en contextos educativos.

3 Denominado en algunos medios como clases virtuales, espacios virtuales enseñanza-aprendizaje, etc. 


\section{LAS PLATAFORMAS VIRTUALES Y LA FACILIDAD DEL APRENDIZAJE VIRTUAL}

Las plataformas virtuales han permitido el desarrollo de enseñanzas educativas por medio de la Web o espacios virtuales dedicados para la educación y transferencia de los conocimientos que incluyen una serie de herramientas que permiten contribuir con la enseñanza de tal forma de comparar dicha transferencia de conocimientos en instrucción presencial (denominado e-learning ${ }^{4}$ ) o una enseñanza mixta (b-learning ${ }^{5}$ ) donde se mezclan la enseñanza y transferencias de conocimientos utilizando el internet con aplicaciones y experiencias iguales o similares a las clases presenciales (Ramboll, et, all, 2004)

El objeto fundamental de dichas plataformas virtuales es la creación de espacios virtuales que permiten la interacción alumno-docente ya sea de manera sincrónica o asincrónica para el desarrollo de los contenidos. Tales espacios virtuales de aprendizaje pueden ser (i): aulas de un centro escolar, (ii) lugares o webs en internet que permiten la enseñanza virtual y se convierte ésta en no presencial, (iii) por último la combinación de las dos características previas lo que convierte la enseñanza en la modalidad de b-learning (Britain S, y Libert, 2004).

La aplicabilidad de dichos modos de transferencia de conocimientos no solo es exclusiva de las IES, sino también de las empresas las cuales lo utilizan para los diversos procesos de capacitación e inducción al recurso humano de sus instituciones. Dicho propósito es ofrecer a su personal una/varias herramienta(s) de perfeccionamiento profesional a bajo coste.

\section{MARCO REFERENCIAL}

Actualmente, las IES están observando cambios de cierta importancia, entre ellos: desplazamiento de procesos de formación e inducción desde los entornos tradicionales a otros entornos (virtuales), otro de los aspectos es la demanda generalizada para que sus estudiantes reciban las competencias, destrezas y habilidades necesarias para la correcta apropiación del aprendizaje continuo, mismo que día a día se vuelve cada vez más dinámico, planificación del conocimiento que genera simultáneamente oportunidades de inserción para

4 Denominada también como una formación a distancia potenciada por la tecnología.(GARCIA, L. 2001)

5 Blended learning, denominado de la forma más sencilla y también la más precisa lo describe como el modo de aprender, el cual combina la enseñanza presencial con la tecnología no presencial: "which combines face-to-face and virtual teaching" (COATEN, 2003; MARSH, 2003). 
nuevos mercados y competencias nuevas en el sector, etc. El entorno de aprendizaje está variando de forma apresurada y las tradicionales IES, ya sean presenciales o a distancia, tienen que reajustar sus sistemas de distribución y comunicación para permitir o facilitar el desarrollo de conocimientos científicos basados en las plataformas de enseñanza virtuales.

El reto no está en reconstruir o reformar el sistema tradicional de clases magistrales, ni alcanzar un aprendizaje completamente independiente vía la web (internet). Lo que hay que hacer es construir o adecuar un nuevo entorno de aprendizaje que progresivamente y sistemáticamente interconecte a sus alumnos unos con otros y con los tutores y profesores y que conecte este aprendizaje con la experiencia que permita tomar ventaja de la abundancia de información y conocimiento accesible mediante las nuevas tecnologías (Hanna, 2000). Además permitir que la integración de las NTIC's en el desarrollo y la práctica educativa perfectamente se puede trascender desde la óptica de un proceso de innovación dentro de las instituciones educativas (Sangrá, 2005).

Revista Economía y Administración (E\&A)

\section{APLICACIÓN DE LAS NTIC'S EN EL PROCESO ENSEÑANZA- APRENDIZAJE}

Las Nuevas Tecnologías de la Información y las Comunicaciones (NTIC's) se han convertido en herramientas orientadas a facilitar la comunicación, e integración del ser humano en actividades cotidianas que permiten la rápida y efectiva comunicación con los diversos entes o agentes económicos sin importar la distancia geográfica que les pueda dividir, permitiendo ello la comunicación y/o transferencia de flujos considerables de insumos convertidos en información necesarios para la toma de decisiones (Gonzales, et, al; 1996).

En el campo educativo dichas NTIC's han logrado el diseño, la creación y mejora continua de procesos de enseñanza-aprendizaje basado en la metodología e-learning o b-learning, logrando con ello la posibilidad de enormes ventajas hasta hace poco tiempo no imaginables.

En la actualidad dichas metodologías de enseñanza-aprendizaje permite la interactuación profesor-alumnos(s) muchas veces de forma concurrente u otras veces de manera asincrónica permitiendo ello el resolver la situación de dificultades particulares de los educandos. Dicha modalidad online permite el desarrollo de técnicas como: un y/o varios alumno(s) apoyándose en el proceso de recuperación de insumos de forma (cliente/proveedor), en donde la relación 
se establece entre alumno(s)-profesor, en donde además dicha comunicación se establece por medio de avisos online (Chat, Msn, etc.) o por medios comunicativos no en tiempo real (e-mail), o el desarrollo de foros y/o conferencias sincronizadas o no, en donde en dicha forma de comunicación todos o la mayoría de participantes deberían tener la oportunidad de participar. (Santagelo, 2003, mencionado en Ferro, et, al). No obstante, hoy por hoy el desarrollo y aplicación de dicho medio necesario e importante para la enseñanza-aprendizaje se va realizando cada ves de manera más frecuente, de forma más rápida y gracias al uso racional de las NTIC's que permite el entorno amigable vuelven efectivo y eficaz la aplicabilidad de tales procesos.

Sin embargo, el mecanismo más extendido y de mayor éxito para el uso de las plataformas e-learning en la enseñanza y el aprendizaje en las IES, en la actualidad, es el campus virtual. Un campus virtual (CV) puede describirse como el lugar dedicado a la enseñanza, aprendizaje, transferencia de conocimientos e investigación formado mediante la convergencia de múltiples aplicaciones de las (NTIC's): internet, la web, comunicación electrónica, video, video-conferencia, multimedia y publicación electrónica (Van Dusen, 1997). Esta definición, ya clásica, puede actualizarse considerando la aparición, posteriormente, de las plataformas e-learning, que integran las herramientas TIC, a las que se refiere Van Dusen, en una única aplicación con fines educativos (inducción y capacitaciones utilizadas en algunos casos en ámbitos laborales). En este momento podemos definir el CV como el espacio en internet creado con aplicaciones web, principalmente plataformas e-learning, con un propósito educativo. Otro término muy relacionado con $\mathrm{CV}$ es el de universidad virtual. Tiene un significado más amplio, ya que se refiere no sólo a los espacios para la enseñanza, aprendizaje e investigación, sino también a los espacios para la administración y organización de todas las actividades y procesos de una universidad (PLS Ramboll, 2004.

En cualquiera de estos casos, las plataformas e-learning componen el soporte técnico de los CV pensados bien como un conjunto de espacios virtuales de enseñanza y aprendizaje de una institución, las IES, o bien como un subconjunto del total de espacios virtuales de esa institución, que están dedicados exclusivamente a la enseñanza y el aprendizaje y la investigación. La finalidad de un CV es que los tutores/profesores y alumnos puedan aprovechar las funciones y bondades que ofrecen para optimizar su trabajo de enseñanza docente.

Revista

Economía y Administración (E\&A) 


\section{VENTAJAS PARA LAS IES LA APLICACIÓN DE LAS NTIC's}

En la actualidad la aplicabilidad de las NTIC's está tomando un auge dinámico y creciente y con ello está permeando una nueva forma de adquisición de conocimientos y logrando además nuevas formas de instrucción (Bartolomé, 1997, mencionado en Ferro, et, al) incidiendo ello en los roles desempeñados por las IES y los educandos en los procesos enseñanza-aprendizaje, en la dinámica de la difusión y diseminación de conocimientos aunque con ello, pasa por el debate de la calidad y/o rigurosidad académica que permita evaluar de manera precisa y rigurosa los conocimientos adquiridos por los educandos bajo esta modalidad.

No obstante, la utilización de las NTIC's, contribuye con múltiples ventajas para la mejora en el desarrollo de contenidos curriculares cada vez de manera más dinámica y frecuente, además contribuye a llevar o desarrollar en la medida de la disponibilidad de infraestructura tecnológica la enseñanza en comunidades inhóspitas logrando con ello cierto reducción de tiempos efectivos para la enseñanza.

Revista Economía y Administración $(E \& A)$

Además se desarrolla de manera substancial la interactuación entre los docentes y los educandos. Aumenta los flujos de colaboración inter unidades académicas de enseñanza, es decir se vuelve necesarios muchas veces la colaboración inter o extra curriculares para lograr colaboración entre unidades inherentes que tengan injerencia en el proceso de preparación académico-curricular de tales cursos o materias de enseñanza.

Por otro lado, el uso e implementación adecuada de las NTIC's en las IES pueden llegar a mejorar la comunicación entre alumnos, contribuyendo con ello en aprendizaje cooperativo al facilitar la organización de actividades grupales (enich y Santos, 2005).

\section{MEJORA DE LA EFICACIA EDUCATIVA}

Quiérase o no, al disponer las IES de la plataforma virtual, adecuada, amigable para el mejoramiento de los métodos en la enseñanza-aprendizaje de los educandos, el proceso de asimilación de nuevos conocimientos se vuelve inherente y se transforma en eficacia en la aplicabilidad de nuevos saberes. No obstante, no se debe dejar desapercibido el grupo de educandos que disponen de alguna desventaja cognitiva en cuanto al manejo en el uso de las NTIC's, además considerar los educandos con limitadas capacidades físicas y psíquicas que les puedan limitar el uso de las herramientas ya que ello posiblemente los 
excluye el uso de tales formas de aprendizaje por lo cual se vuelve pertinente y oportuno el desarrollo de formas alternativas de enseñanza-aprendizaje que permitan solventar dichas dificultades para los educandos. (Soto y Fernández, 2003, mencionado en Ferro, et, al).

Asimismo, el avance en el desarrollo e implementación de las NTIC's en la educación supone la obligación de concientizar y estimular en los estudiantes las capacidades de analizar sobre la enorme cantidad de información disponible, la propagación de los medios de comunicación masiva (Televisión, Internet), los cuales se han convertido en un espacio de interacción social, el debilitamiento de las barreras geográficas en la medida que los medios de comunicación (correo electrónico o teléfono) permiten conectarse con cualquier persona en el mundo, la concentración de la producción de información en unos pocos. Además, el potencial de interactividad, a diferencia de la televisión o el radio, permite que los usuarios no sólo sean consumidores sino generadores de información. Sin embargo se ha sembrado también un gran germen de desigualdad puesto que, aunque las tecnologías ofrecen oportunidades, éstas no se distribuyen homogéneamente en la población, reconociendo el fenómeno de la brecha digital como una nueva forma de marginación social. Internet es un espacio de expresión de la sociedad, en este medio se reflejan las actitudes, creencias y valores de grupos sociales y culturales; donde también forman parte de ella los prejuicios, los abusos, insolencias o crímenes.

\section{VENTAJAS ACADÉMICAS PARA EL DOCENTE}

Las NTIC's como herramienta para la transferencia de conocimientos y aprendizaje significativo sirve como instrumento docente, permitiendo al tutor reorientar y planificar de manera más eficiente su tiempo y estimulando en el educando el desarrollo de las facultades cognitivas superiores (Mata, 2002; Martínez et al., 2003). Además, facilitan la actualización profesional del profesor hasta determinado punto de manera más fácil y rápida, debido a que con el acceso al Internet encuentran cursos y capacitaciones online (virtuales) que pueden contribuir a mejorar sus competencias profesionales sin necesidad, alguna veces de moverse de su cubículo de trabajo. Asimismo, contribuyen al desarrollo de la práctica en forma sistemática mediante ejercicios auto correctivos de refuerzo sobre técnicas instrumentales o presentación de conocimientos generales (Coll et al., 2007, mencionado en Ferro, et, al), liberándole de tareas repetitivas, monótonas y en algunos casos rutinarias, toda vez que mejoran la evaluación y el control del estudiante. 


\section{ACTIVIDADES COMPLEMENTARIAS DE APOYO AL APRENDIZAJE}

Las NTIC's utilizadas de forma racional y responsable pueden llegar a ser sumamente útiles para realizar actividades que puedan ser complementarias en el proceso de aprendizaje y de obtención de algunos insumos en las que los estudiantes pueden auto controlar su trabajo. El acceso efectivo/eficaz a múltiples recursos educativos y entornos de aprendizaje, pone al alcance de los educandos todo tipo de información y múltiples recursos didácticos digitales, dispuestos en formatos de CD/DVD e Internet, que fortalecen y complementan los procesos de enseñanza-aprendizaje.

Asimismo, las tareas educativas realizadas con el ordenador y con el acceso a los insumos disponibles en el Internet permiten al educando obtener un alto grado de interdisciplinariedad. También, debido a la versatilidad y gran capacidad de almacenamiento de los ordenadores, es fácil realizar muy diversos tipos de tratamiento/manipulación de información a una información muy amplia y variada.

Revista

Economía y

Administración

(E\&A)

\section{PANORAMA DE INTEGRACIÓN DE LAS NTIC'S EN LA EDUCACIÓN}

Las transformaciones educativas, siendo estas del nivel de educación media, y del nivel superior deberá crear conciencia en la población de forma tal que pueda crear mayores niveles de conciencia y formación que permita a los educandos aplicar el conocimiento de para que éste pueda generar soluciones de manera expedita a la problemáticas de los contextos que corresponde.

Además, la integración de las NTIC's en la ES, deberán concebir la posibilidad de aspectos que pueden incidir para que los cambios incluyan elementos que puedan afectar el desarrollo adecuado del proceso enseñanza-aprendizaje. Así pues las implicaciones que pueden surgir se centran en varios aspectos, mismos que se describen a continuación:

a. Deberá enfocarse una ES que considere una educación holística, es decir que los conocimientos brindados a la fuerza de trabajo considere aspectos clave que permitan a dicha fuerza laboral integrarse de manera expedita considerando fortalecer dichos programas académicos en el desarrollo 
de habilidades y destrezas que el proceso de globalización tecnológica pueda concebir, se deberá disponerse desde las IES de conocimientos dinámicos, agiles, que aunado a políticas públicas-privadas consideren la inversión en educación necesaria para el desarrollo e innovación.

b. Enfocarse y brindarse énfasis en el aprendizaje significativo que permita al educando desenvolverse de manera eficaz y eficiente en un ambiente de trabajo que esté acorde al proceso globalizante.

c. Educación para todos, ello ajustándose a los resultados considerando las diversas conferencias educativas llevadas a cabo a nivel global, en donde se ha enfocado y orientado a que se estime pertinente el individuo como un actor social, el cual es el sujeto del desarrollo.

d. La educación por medio de las NTIC's deberán estar disponibles durante todo el tiempo de desarrollo del proceso académico-enseñanza de los educandos, etc.

e. Empoderamiento de los profesores, ello implica que el recurso humano deberá brindársele la importancia en dicho proceso, ello debido a que ése es un elemento esencial en el proceso aprendizaje-enseñanza que estará en contacto directo con los educandos, el cual será el vehículo por el cual deberán fluir los conocimientos dispuestos en las herramientas tecnológicas que deban ser asimilados y comprendidos de manera expedita por los estudiantes.

f. Preparación inicial de parte de los profesores, ya que ello es vital en el proceso enseñanza aprendizaje, la cual brindará a los tutores el domino y fortalezas de la enseñanza aplicando las NTIC's.

Tal integración en los espacios educativos puede observarse (gráfico 1) muestra el lugar de acceso a Internet de niños y jóvenes de entre 10 y 19 años, según quintil de ingresos. El principal lugar de acceso a Internet para los sectores sociales más pobres es la escuela, en una proporción que varía de acuerdo al país. En el Ecuador y Panamá, se aprecia esta situación claramente: cerca del 60\% del quintil inferior accede a Internet en establecimientos educativos y apenas un mínimo porcentaje lo hace en el hogar. Por lo tanto, la introducción de las TIC en la educación contribuye a los procesos de integración social, reduciendo la polarización y la reafirmación de las inequidades prexistentes. A esto se debe la importancia que se ha atribuido en la región al sector educativo como un espacio para la reducción de las brechas sociales. 
Revista Economía y Administración $(E \& A)$
AMÉRICA LATINA Y EL CARIBE (PAÍSES SELECCIONADOS): ACCESO A INTERNET DE NIÑOS Y JOVENES SEGÚN LUGAR Y QUINTIL DE INGRESO (En porcentajes)

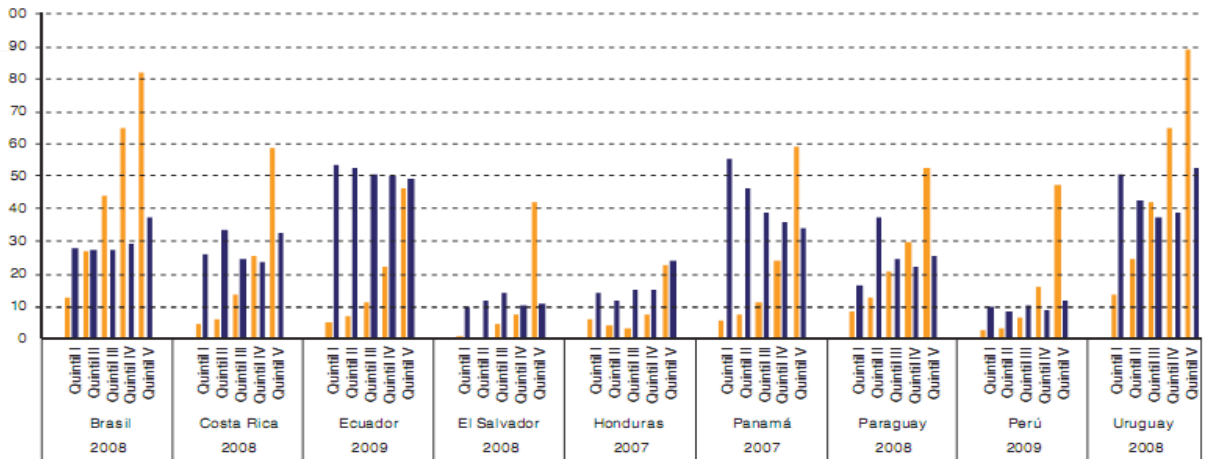

Gráfico No. 1. América Latina y el Caribe (Países seleccionados): acceso a Internet de niños y jóvenes según lugar y quintil de ingreso (en porcentajes)

Fuente: Comisión Económica para América Latina y el Caribe/Observatorio para la Sociedad de la Información en Latinoamérica y el Caribe (CEPAL/OSILAC), sobre la de encuestas de hogares de los respectivos países. (Cálculo realizado sobre el número total de usuarios de Internet de entre 10 y 19 años de cada país, según quintil de ingreso.)

Además de la aplicación de las NTIC's en los ámbitos educativos no deja de ser determinante el uso de tales herramientas para la correcta aplicación que permitan eficientar y/o mejorar la gestión de la buena gobernanza reduciendo hasta cierto punto los procesos burocráticos, tal acción puede observarse por medio de algunos resultados hacia la gobernabilidad.

\section{RESULTADOS ORIENTADOS A LA BUENA GOBERNANZA}

Otro de los sectores que pueden llegar a beneficiarse del acceso a las NTIC's en el proceso de educación superior trasciende las fronteras del aspecto educativo y logra superar e incidir en aspectos de gobiernos electrónicos, incidir en mejorar o aumentar el PIB per cápita de las poblaciones, mismas que requieren que el personal en las diversas áreas de ocupaciones dispongan de las habilidades y destrezas académicas-tecnológicas para desarrollar con buen suceso tales acometidos (Gráfico 2). 


\section{ÍNDICE DE GOBIERNO ELECTRÓNICO Y PIB PER CÁPITA}

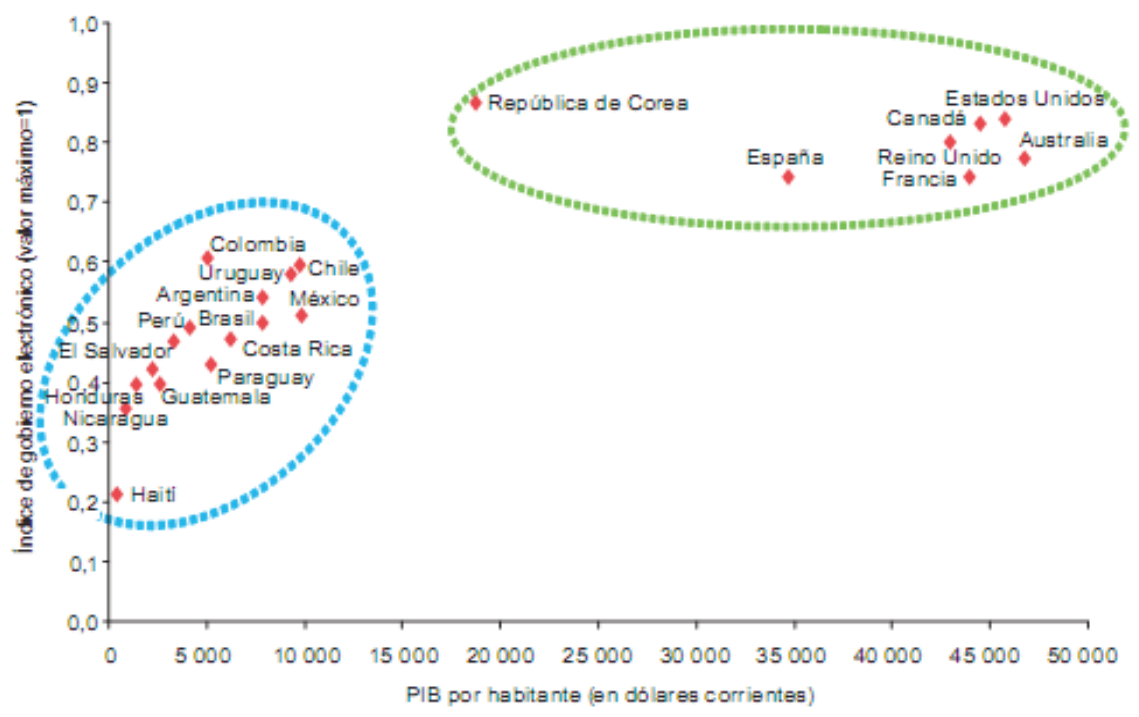

Gráfico No. 2. Índice de Gobierno electrónico y PIB Per cápita

Fuente: Comisión Económica para América Latina y el Caribe (CEPAL), sobre la base de Naciones Unidas The United Nations e-government Survey 2010 (ST/ESA/PAD/SER.E/131), Nueva York, 2010. Publicación de las Naciones Unidas, $N^{o}$ de venta: E.10.II.H.2; y Banco

\section{CONSIDERACIONES FINALES}

El uso de las NTIC's en el proceso de enseñanza-aprendizaje en la educación superior enmarcado en el nuevo contexto del proceso de globalización resulta oportuno, puesto que permite la realización de diferentes tipos de funciones que van desde el acceso e intercambio de información, hasta la creación de entornos simulados que facilitan la realización de prácticas de fácil control y preparación por los docentes. Además, su carácter flexible, dinámico y abierto hace que puedan ser utilizadas en diferentes contextos y situaciones de aprendizaje, desde la transmisión de información, hasta la simulación de fenómenos o la realización de ejercicios, la evaluación de los conocimientos y habilidades o la tutorización. 
Sin embargo, la principal ventaja de estas tecnologías recae sobre la posibilidad de romper las barreras espacio y barreras temporales que han influido sobre las actividades formativas en los sistemas educativos universitarios convencionales. El ciberespacio ha creado entornos virtuales de aprendizaje donde el espacio educativo no reside en ningún lugar concreto, la educación es posible sin límites temporales y la interactividad entre los agentes implicados tiene lugar sin limitaciones de espacio ni de tiempo.

Las NTIC's también permiten una interacción sujeto-máquina y la adaptación de ésta a las características educativas y cognitivas de los educandos. De esta forma, los estudiantes dejan de ser meros receptores pasivos de información, pasando a ser procesadores activos y conscientes de la misma.

Asimismo, resulta curioso que la ventaja menos valorada en torno al uso de las NTIC's sea el ahorro de tiempo que éstas deberían proporcionar al profesor para dedicarlo a otras tareas. No en vano, existen voces discordantes al momento de considerar el aspecto temporal como una ventaja, al afirmar que su uso requiere mucho más tiempo del profesor que los medios convencionales: cursos de

Revista Economía y Administración (E\&A) alfabetización, tutorías virtuales, gestión y administración del correo electrónico personal, búsqueda de información en Internet, etc.

Además, este no sería el único inconveniente atribuible al uso de las NTIC's en el proceso de enseñanza-aprendizaje universitario. Otros como el considerable aumento de informaciones no fiables, diversas dificultades para localizar, seleccionar y analizar los insumos, los riesgos de ansiedad o adicción de los estudiantes por la continua interacción con el ordenador; los problemas de falta de sociabilidad o la aparición de dolencias (cansancio visual y otros problemas físicos provocados por malas posturas producidas $\mathrm{u}$ ocasionadas por un exceso de tiempo trabajando ante el ordenador), son algunos de los ejemplos de la otra cara de la moneda que debe ser objeto de análisis para conducir y para reflexionar detenidamente y hacer un balance responsable de la situación, para que el resultado neto de su generalización en el contexto resulte positivo. 


\section{BIBLIOGRAFÍA}

Britain S, y Libert, 2004). Britain, S. y Liber, O., 2004. "A Framework for the Pedagogical Evaluation of eLearning Environment". JISC-commissioned report, consultado mayo 2012, desde http://www.cetis.ac.uk/members/pedagogy/ files/4thMeet_framework/VLEfullReport.

Cenich,G. y Santos, G., 2005. "Propuesta de aprendizaje basado en proyectos y trabajo colaborativo: experiencia de un curso en línea". Revista Electrónica de Investigación Educativa. Vol.7, No 2, http://redie.uabc.mx/vol7no2/contenido-cenich.html.

Ferro, Carlos; Martínez, Ana Isabel; Otero, Ma Carmen, 2009. "Ventajas del uso de las TICs en el proceso de enseñanza aprendizaje desde la óptica de los docentes universitarios españoles”. EDUTEC, Revista Electrónica de Tecnología Educativa. Núm. 29/ Julio 2009.

González, Ángel; Gisbert, Mercé; Guillem, Antoni; Jiménez, Bonifacio; LLADÓ, Fátima y Rallo, Robert (1996). "Las nuevas tecnologías en la educación, Editorial Salinas.

Jesús et al. (eds.). Redes de comunicación, redes de aprendizaje. Universitat de les Illes Balears: EDUTEC'95, págs. 409-422.

Lanza, Mario, 2007. "Las TIC en la Educación de Centroamérica, adopción de las tecnologías de la información y comunicación en los procesos de enseñanza-aprendizaje", Banco Centroamericano de Integración Económica (BCIE), Tegucigalpa, Honduras.

Ramboll Management, 2004. Studies in the Context of the E-learning Initiative: Virtual Models of European Universities (Lot). Draft Final Report to the EU Commission, DG Education y Culture". descargado en abril 2012, http://www. elearningeuropa.info/extras/pdf/virtual_models.pdf.

Rogers, 2003. Diffusion of innovations $5^{\mathrm{a}}$ Ed. Free Press, New York.

Sangrá, 2005. E-Learning, nuevos modelos de aprendizaje y calidad: ¿dónde está la innovación? Isla verde. Puerto Rico En actas de 4to congreso de Web e-educación

Santángelo,Horacio Néstor (2003). "Modelos pedagógicos en los sistemas de enseñanza no presencial basados en nuevas tecnologías y redes de comunicación". En Martínez Sánchez, Francisco (coord.):Redes de comunicación en la enseñanza: Las Nuevas Perspectivas del Trabajo Corporativo. Barcelona: Paidós Ibérica,

- $\quad$ Shea, Pickett and Li, C.S., 2005. Increasing access to higher education; a study of the diffusion of online teaching among 913 college faculty. International review of research in open and distance learning.

- Van Dusen, G. C., 1997. "The Virtual Campus. Technology and Reform in Higher Education”. En ASHE-ERIC Higher Education Report 25. 